\title{
Long-Term Effects of Bone Marrow-Derived Mesenchymal Stem Cells in Dextran Sulfate Sodium-Induced Murine Chronic Colitis
}

Hyun Jung Lee, Sun-Hee Oh, Hui Won Jang, Ji-Hee Kwon, Kyoung Jin Lee, Chung Hee Kim, Soo Jung Park, Sung Pil Hong, Jae Hee Cheon, Tae II Kim, and Won Ho Kim

Department of Internal Medicine and Institute of Gastroenterology, Yonsei University College of Medicine, Seoul, Korea

Background/Aims: Bone marrow-derived mesenchymal stem cells (BM-MSCs) have shown beneficial effects in experimental colitis models, but the underlying mechanisms are not fully understood. We investigated the long-term effects of BM-MSCs, particularly in mice with chronic colitis. Methods: Chronic colitis was induced by administering 3\% dextran sulfate sodium (DSS) in a series of three cycles. BMMSCs were injected intravenously into DSS-treated mice three times during the first cycle. On day 33, the therapeutic effects were evaluated with clinicopathologic profiles and histological scoring. Inflammatory mediators were measured with real-time polymerase chain reaction. Results: Systemic infusion of BM-MSCs ameliorated the severity of colitis, and body weight restoration was significantly promoted in the BMMSC-treated mice. In addition, BM-MSC treatment showed a sustained beneficial effect throughout the three cycles. Microscopic examination revealed that the mice treated with BM-MSCs had fewer inflammatory infiltrates, a lesser extent of inflammation, and less crypt structure damage compared with mice with DSS-induced colitis. Anti-inflammatory cytokine levels of interleukin-10 were significantly increased in the inflamed colons of BM-MSC-treated mice compared with DSS-induced colitis mice. Conclusions: Systemic infusion of BM-MSCs at the onset of disease exerted preventive and rapid recovery effects, with long-term immunosuppressive action in mice with repeated DSS-induced chronic colitis. (Gut Liver 2016;10:412-419)

Key Words: Bone marrow; Mesenchymal stem cell; Dextran sulfate sodium; Chronic colitis; Inflammatory bowel disease

\section{INTRODUCTION}

Inflammatory bowel disease (IBD), ulcerative colitis (UC) and Crohn's disease (CD), are chronic relapsing inflammatory disorders of the gastrointestinal tract. Although the pathogenesis of UC and CD is still unclear, growing evidence has shown that epithelial barrier defects and dysregulated immune system reactions in genetically susceptible individuals contribute to sustained mucosal inflammation. ${ }^{1,2}$ An inappropriate adaptive immune response activated by commensal microbes is known to directly cause tissue damage, and recent studies report that clearance of over-reactive or autoreactive T-cells and balance of regulatory and effector T cells (Th1 and Th2) are disturbed in patients with $\mathrm{IBD}^{2,3}$ As for the treatment of IBD, because the need for bowel resection due to failure of medical treatments is remained yet, there is an unmet demand for new therapeutic approaches targeting uncontrolled inflammatory activity to complement the limited efficacy of current immunosuppressive and biologic agents. ${ }^{4-6}$

Candidates for new alternative treatments include cell therapy. The first use of hematopoietic stem cells (HSC) as a treatment for IBD was reported in patients with $\mathrm{CD}$, who remained in remission after an HSC transplant for non-Hodgkin's lymphoma. ${ }^{7}$ With the development of autologous bone marrow (BM) stem cell transplantation for $\mathrm{CD},{ }^{8}$ mesenchymal stem cells (MSCs) have been also reported as an effective source of cell therapy.

MSCs, derived from various tissues like BM and adipose tissue, are a heterogeneous population of stromal stem cells that can self-renew and differentiate into different cell types, such as bone, cartilage, and fat cells. ${ }^{9}$ In addition to their ability to promote tissue regeneration from damaged tissue progenitors, ${ }^{9,10}$ MSCs have been shown to regulate both innate and adaptive

\footnotetext{
Correspondence to: Tae Il Kim

Division of Gastroenterology, Department of Internal Medicine, Yonsei University College of Medicine, 50 Yonseiro, Seodaemun-gu, Seoul 03722, Korea

Tel: +82-2-2228-1965, Fax: +82-2-393-6884, E-mail: taeilkim@yuhs.ac

Received on May 20, 2015. Revised on July 16, 2015. Accepted on July 16, 2015. pISSN 1976-2283 eISSN 2005-1212 http://dx.doi.org/10.5009/gnl15229

(c) This is an Open Access article distributed under the terms of the Creative Commons Attribution Non-Commercial License (http://creativecommons.org/licenses/by-nc/4.0) which permits unrestricted non-commercial use, distribution, and reproduction in any medium, provided the original work is properly cited.
} 
immune responses by inhibiting $\mathrm{T}$ cell proliferation, B cell function, and dendritic cell maturation. ${ }^{10-12}$ Therefore, MSCs are emerging as a candidate for treatment of immune-mediated disease, including IBD. Indeed, an in vivo immunosuppressive effect was reported in $94 \%$ of patients with acute, severe graftversus-host disease who were treated with intravenous infusions of MSCs. ${ }^{13,14}$ In addition, with beneficial effect of MSCs in experimental colitis models, ${ }^{15-17}$ some clinical trials demonstrated positive results of MSCs therapy in Crohn's perianal fistula and refractory luminal CD. ${ }^{18,19}$ However, most experiments have focused on the prevention and improvement of inflammation in a relatively short period after injection of MSCs and the underlying mechanisms for the beneficial effects of MSCs are not yet fully understood. Therefore, we investigated the long-term effect of BM-MSCs in mice with chronic colitis induced by repeated administration of dextran-sulfate sodium (DSS).

\section{MATERIALS AND METHODS}

\section{Animals}

Female C57BL/6 mice (aged 8 weeks, weighing 17 to $21 \mathrm{~g}$ ) were purchased from The Jackson Laboratory (Bar Harbor, ME, USA; http://www.jax.org). Mice were housed under specific pathogen-free conditions in a controlled temperature $\left(24^{\circ} \mathrm{C}\right)$ and 12-12 hour light-dark cycle and provided standard diet and water ad libitum. All animal experiments were approved by the Yonsei University Institutional Animal Care and Use Committee (approval number: 09-093-3) and performed according to the Guide for the Care and Use of Laboratory Animal (National Research Council, USA, 2010).

\section{BM-MSCs isolation and culture}

Green fluorescent protein-transgenic (GFP-Tg) C57BL/6 mouse BM-MSCs were isolated and cultured as described previously. ${ }^{20}$ The BM cells were obtained by flushing the femurs, tibias, and iliac crests from 6-week-old mice with phosphate buffered saline (PBS) supplemented with 2\% fetal bovine serum (FBS; HyClone Laboratories Inc., Logan, UT, USA). The collected cells were cultured and maintained in a $75-\mathrm{cm}^{2}$ culture flask containing Dulbecco's modified Eagle medium (HyClone Laboratories Inc.) supplemented with 10\% FBS, $100 \mathrm{U} / \mathrm{mL}$ penicillin (HyClone Laboratories Inc.), and $100 \mathrm{~g} / \mathrm{mL}$ streptomycin (HyClone Laboratories Inc.). Nonadherent cells were removed after 3 days, and only adherent cells were maintained in culture for 2 to 3 weeks until almost confluent. Then, adherent cells were detached using 0.25\% trypsin-ethylenediaminetetraacetic acid (HyClone Laboratories Inc.). Subsequent passaging and seeding of the cells was performed at a density of $1 \times 10^{7}$ cells.

\section{Colitis induction and experimental design}

Chronic colitis was induced by administering 3\% (w/v) DSS (molecular weight: 36,000 to 50,000 Da; MP Biochemicals,
Santa Ana, CA, USA) via drinking water in a cyclic manner, which consisted of three cycles (4 days/cycle) of DSS and 6 days of normal drinking water ad libitum. ${ }^{21}$ GFP-Tg mice BM-MSCs $\left(1 \times 10^{7}\right.$ cells $\left./ 200 \mu \mathrm{L}\right)$ were injected intravenously into the DSStreated mice $(n=5)$ on days 1,3 , and 5 during the first cycle (BM-MSC-treated group). Dosage and frequency of BM-MSCs were determined on the basis of our preliminary data and previous studies. ${ }^{16,17}$ In the control group, DSS-treated mice received PBS $(n=5)$. All mice were sacrificed at day 33 after being fed DSS water, and the colon was processed for histological analysis or frozen in liquid nitrogen for RNA extraction. The therapeutic effect of BM-MSC treatment was evaluated by clinicopathologic profiles of body weight, colon length, and histological scoring.

\section{Histological analysis}

The entire colon was removed from the cecum to the anus, fixed in 4\% paraformaldehyde, embedded in paraffin, and sliced into 4- $\mu \mathrm{m}$ sections. After hematoxylin and eosin (H\&E) staining, histological analysis was performed in a blind manner. The following three parameters were studied as described previously: ${ }^{22}$ inflammation severity $(0=$ none, $1=$ mild, $2=$ moderate, and $3=$ se vere), inflammation extent ( $0=$ none, $1=$ mucosa, $2=$ mucosal and submucosa, and $3=$ transmural), and crypt damage $(0=$ none, $1=$ basal $1 / 3$ damaged, $2=$ basal $2 / 3$ damaged, $3=$ crypts loss, but surface epithelium present, 4=both crypts and surface epithelium loss). Total histological score was defined as the sum of the three parameters.

Immunohistochemical (IHC) staining for GFP, using a Vecastain $\mathrm{ABC}$ kit (Vector Laboratories, Burlingame, CA, USA), was performed to identify the in vivo localization of transplanted BM-MSCs in the inflamed colon. Colonic tissue samples were incubated first with the primary anti-GFP antibody (Abcam, Cambridge, MA, USA) overnight at $4^{\circ} \mathrm{C}$, then with a biotinylated secondary linking antibody, and finally with a streptavidinperoxidase complex for 1 hour. The final color product was developed using aminoethylcarbazole (Dako, Glostrup, Denmark). Sections were counterstained with hematoxylin, and tissues were photographed using an Olympus photomicroscope (Olympus Corp., Tokyo, Japan).

\section{Cytokine measurement}

mRNA expression of inflammatory mediators, such as IL10 , transforming growth factor $\beta$ (TGF- $\beta$ ), and tumor necrosis factor $\alpha$ (TNF- $\alpha$ ), in the inflamed colon was measured by realtime polymerase chain reaction (PCR). Isolated colons were frozen in liquid nitrogen and stored at $-70^{\circ} \mathrm{C}$ until use. Tissue samples were homogenized using a Polytron ${ }^{\circledR}$ System PT1200E (Kinematica AG, Luzern, Switzerland). Total RNA was extracted from the colonic tissues using a miRNeasy ${ }^{\circledR}$ Mini Kit (QIAGEN, Hilden, Germany) according to the manufacturer's instructions and then reverse transcribed using the PrimerScript ${ }^{\mathrm{TM}}$ RT Master Mix (Takara, Kusatsu, Japan). Template DNA was then amplified 
by PCR using the Applied Biosystems (Foster City, CA, USA) StepOnePlus ${ }^{\mathrm{TM}}$ real-time PCR system and the following primers: 5'-GGT TGC CAA GCC TTA TCG GA-3' (IL-10 sense primer), 5'ACC TGC TCC ACT GCC TTG CT-3' (IL-10 antisense primer), 5'TGA CGT CAC TGG AGT TGT ACG G-3' (TGF- $\beta$ sense primer), 5'-GGT TCA TGT CAT GGA TGG TGC-3' (TGF- $\beta$ antisense primer), 5'-CAT CTT CTC AAA ATT CGA GTG ACA A-3' (TNF- $\alpha$ sense primer), 5'-TGG GAG TAG ACA AGG TAC AAC CC-3' (TNF- $\alpha$ antisense primer), 5'-CGC GGT TCT ATT TTG TTG GT3' (Rn18S sense primer), 5'-AGT CGG CAT CGT TTA TGG TC3' (Rn18S antisense primer). Thermal cycling conditions were 30 seconds at $95^{\circ} \mathrm{C}$, followed by 40 cycles of 5 seconds at $95^{\circ} \mathrm{C}$, 34 seconds at $60^{\circ} \mathrm{C}$, and 15 seconds at $95^{\circ} \mathrm{C}$. For relative quantification, we compared the amount of target normalized to the Rn18S amplification.

\section{Statistical analysis}

Data are expressed as means \pm standard error of mean (SEM). Parametric and nonparametric analyses were performed using the Student t-test and Mann-Whitney U test, respectively. A value of $p<0.05$ was considered statistically significant for all tests. All statistical analyses were performed using SPSS version 20.0 for Windows (IBM Corp., Armonk, NY, USA).

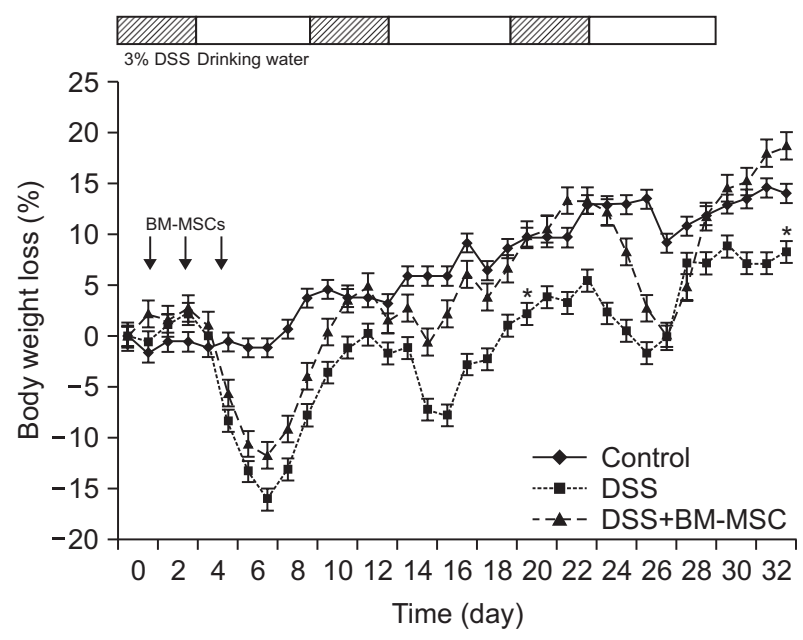

Fig. 1. Clinical effects of bone marrow-derived mesenchymal stem cells (BM-MSCs) in repeated dextran sulfate sodium (DSS)-induced chronic colitis. Mice ( $\mathrm{n}=5$ /group) received 3\% dextran sulfate sodium (DSS) in their drinking water in a cyclic manner consisting of three cycles of DSS for 4 days/cycle and 6 days of normal water. Green fluorescent protein-transgenic mouse BM-MSCs were injected intravenously on days 1,3 , and 5 during the first cycle. DSS- and BMMSCs-treated mice showed a significant increase in body weight compared with DSS-only-treated mice. The data are presented as the mean \pm standard error of mean. ${ }^{*} p<0.05$ vs $3 \%$ DSS+BM-MSCs.

\section{RESULTS}

1. BM-MSCs injection induced prevention and rapid recovery of weight loss in DSS-induced chronic colitis

We first investigated the clinical therapeutic efficacy of BMMSCs in a DSS-induced colitis model. Administration of 3\% DSS in three cycles resulted in three peaks of colitis that were characterized by substantial weight loss and bloody diarrhea compared with the normal, untreated mice. However, DSStreated mice injected with BM-MSCs (BM-MSC-treated group) showed less weight loss than mice in the DSS-treated only group. Furthermore, body weight restoration was significantly promoted in BM-MSC-treated mice compared with the DSStreated only group. Significant differences were noted at days 20 and 33 of the recovery periods of each DSS administration cycle (Fig. 1). In particular, regarding the course of weight change, BM-MSC injection in the first cycle of DSS administration showed a preventive effect on weight loss in the second cycle, and rapid restoration of weight loss rather than a preventive effect in the third cycle, compared with the untreated DSSinduced colitis group (Fig. 1).

Macroscopic examination also revealed that entire colons from the DSS-treated mice were short, hyperemic, and edematous, indicating chronic inflammation in the colon. As shown in Fig. 2, BM-MSC treatment appeared to attenuate the colon shortening, though not to a statistically significant extent.

In summary, injection of BM-MSCs in the first cycle showed a sustained beneficial effect throughout the three cycles of DSS administration.

\section{BM-MSCs injection showed sustained histologic im- provement in DSS-induced chronic colitis}

We sacrificed mice on day 33 for further evaluation of colon inflammation and crypt damage and microscopic evaluation of the long-term, anti-inflammatory effect of BM-MSCs. Colon tissue samples were microscopically examined following H\&E staining. DSS-treated mice that were not treated with BM-MSCs showed severe infiltration of inflammatory cells and disruption of crypt architecture. BM-MSCs treatment of mice with DSSinduced colitis exhibited a protective effect against DSS-induced histological damage, including fewer inflammatory infiltrates (less severe inflammation), a lower extent of inflammation, and less crypt structure damage, compared with untreated DSSinduced colitis mice. DSS-treated mice transplanted with BMMSCs displayed significant reduction of total histological scores compared with scores for the DSS-treated only group (Fig. 3).

The IHC staining performed to identify transplanted BMMSCs in the colon on day 33 revealed no definite GFP positive cell in the colons of BM-MSC-injected chronic colitis mice (data not shown). 


\section{A}

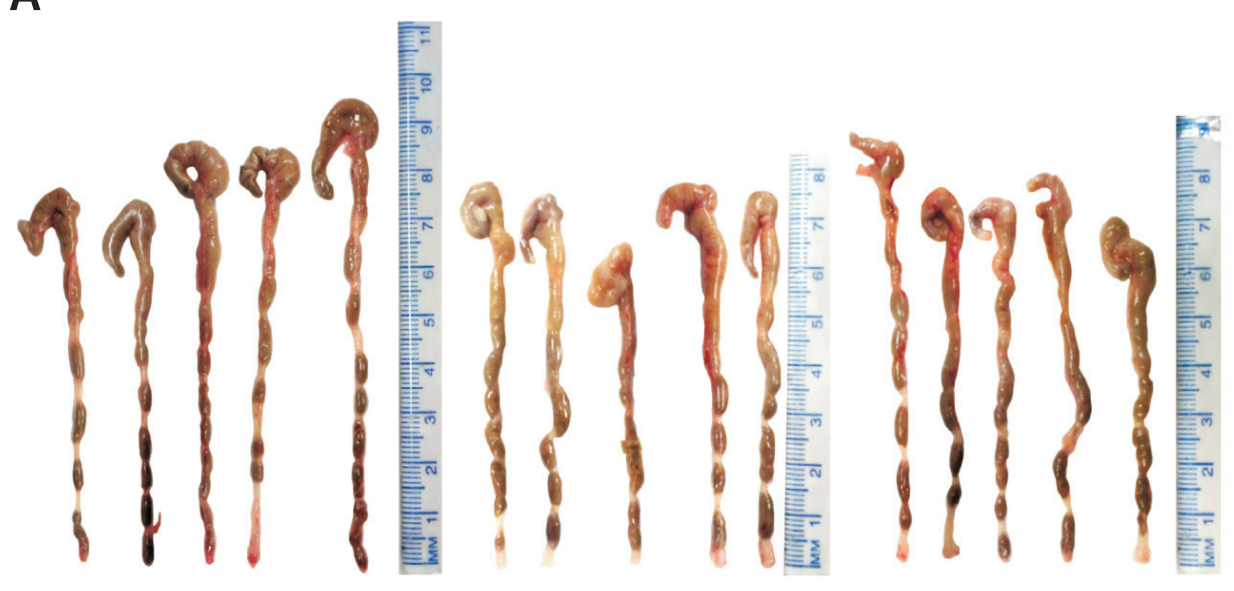

B

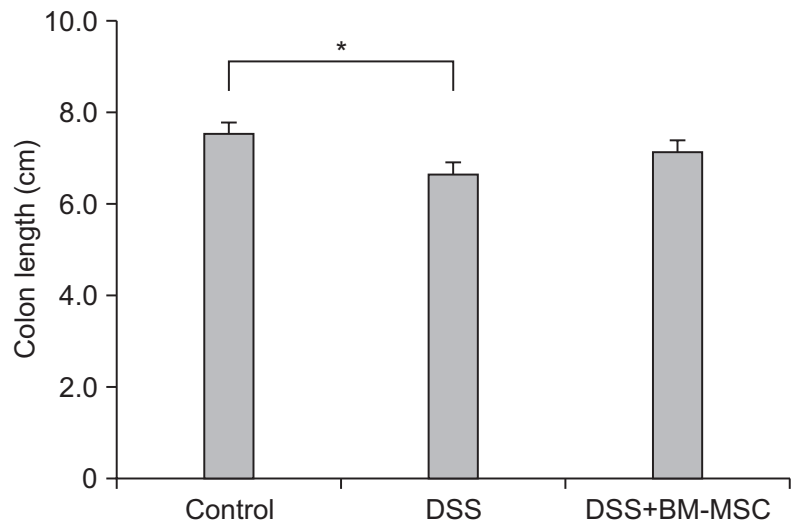

3. BM-MSCs injection maintained anti-inflammatory cytokine response in DSS-induced chronic colitis

To identify the underlying mechanisms for the beneficial effect of BM-MSCs in DSS-induced chronic colitis, we investigated the influence of BM-MSC transplantation on pro- and anti-inflammatory cytokine production. Real-time PCR analysis of colonic tissues showed that mRNA expression of TNF- $\alpha$ was significantly increased in DSS-treated only mice and decreased in BM-MSC-treated mice with DSS-induced colitis. In contrast, mRNA expression of IL-10 and TGF- $\beta$ was increased in the inflamed colons of BM-MSC-injected mice compared with that in colon samples of DSS-treated only mice. Notably, IL-10 production, which is known to act as an anti-inflammatory regulatory cytokine, ${ }^{23,24}$ was significantly upregulated by about 10 -fold in BM-MSC-treated mice, compared with DSS-treated only mice (Fig. 4).

\section{DISCUSSION}

In the present study, we identified the long-term beneficial role of BM-MSCs in the improvement of clinicopathologic activity in a DSS-induced chronic colitis mouse model. Remark-
Fig. 2. Effects of bone marrow-derived mesenchymal stem cells (BMMSCs) on macroscopic findings (A) and colon length (B). On day 33, the mice were sacrificed and their colons were removed from the cecum to the anus. The BM-MSC-treated, dextran-sulfate sodium (DSS)-induced colitis group showed a trend toward a lesser decrease in colon length compared with the DSS-treated group, but the difference did not reach statistical significance (control, untreated group $7.5 \pm 0.6 ; 3 \%$ DSS-treated group $6.6 \pm 0.7 ; 3 \%$ DSS+BM-MSC-treated group $7.1 \pm 0.5$ ). The colon length is presented as the mean \pm standard error of mean ( $* 0.05)$.

ably, initial injection of BM-MSCs at disease onset manifested a sustained beneficial effect throughout three cycles of DSS administration. Immunological assays indicated that the effect of BM-MSCs could be mediated by up-regulation of antiinflammatory cytokine, IL-10.

Due to their immunoregulatory function and differentiation potential, BM-MSCs are increasingly being used to treat autoimmune and systemic inflammatory disease. BM-MSCs are known to be immunoprivileged because of their low expression of class II major histocompatibility complex (MHC-II) and costimulatory molecules in their cell surface, which makes them invisible to the immune system. ${ }^{10}$ Previous studies report that co-culture with MSCs influences cytokine production of T-cell subsets, which results in suppression of T-cell effector function (by decreasing levels of pro-inflammatory cytokines such as TNF- $\alpha$, IFN- $\gamma$, IL-6, and IL-17) and enhancement of T-cell suppressor functions (by increasing IL-10 and TGF- $\beta$ levels). ${ }^{25,26}$ Similarly, because IBD is related to immune dysregulation, recent studies have considered immunomodulatory cytokines as therapeutic targets ${ }^{4,27}$ and BM-MSCs are emerging as one of the promising therapies for IBD. However, though accumulating data have shown beneficial effects of BM-MSCs in an experimental colitis model, ${ }^{15,17,28,29}$ the mechanisms are yet to 

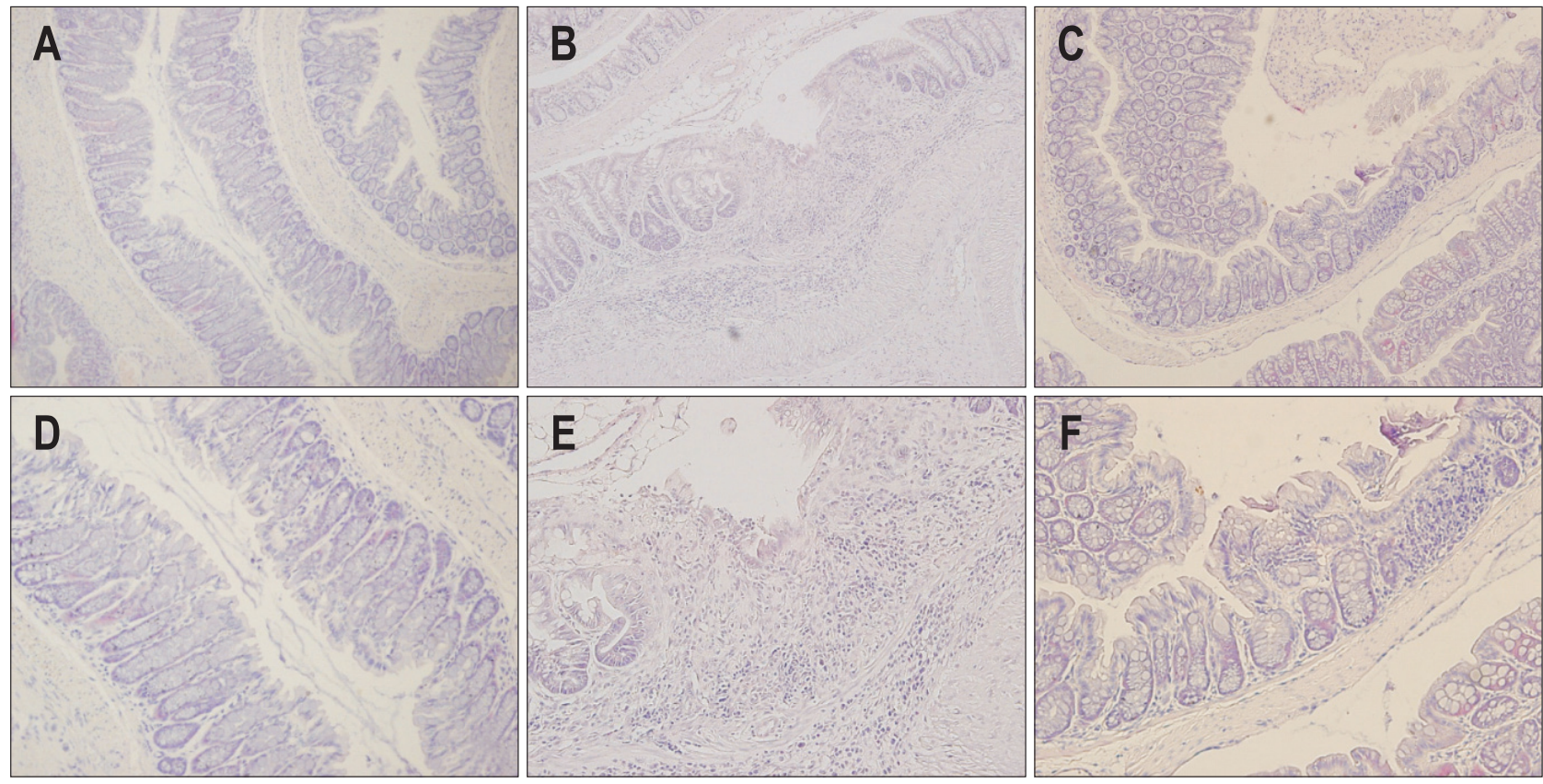

G
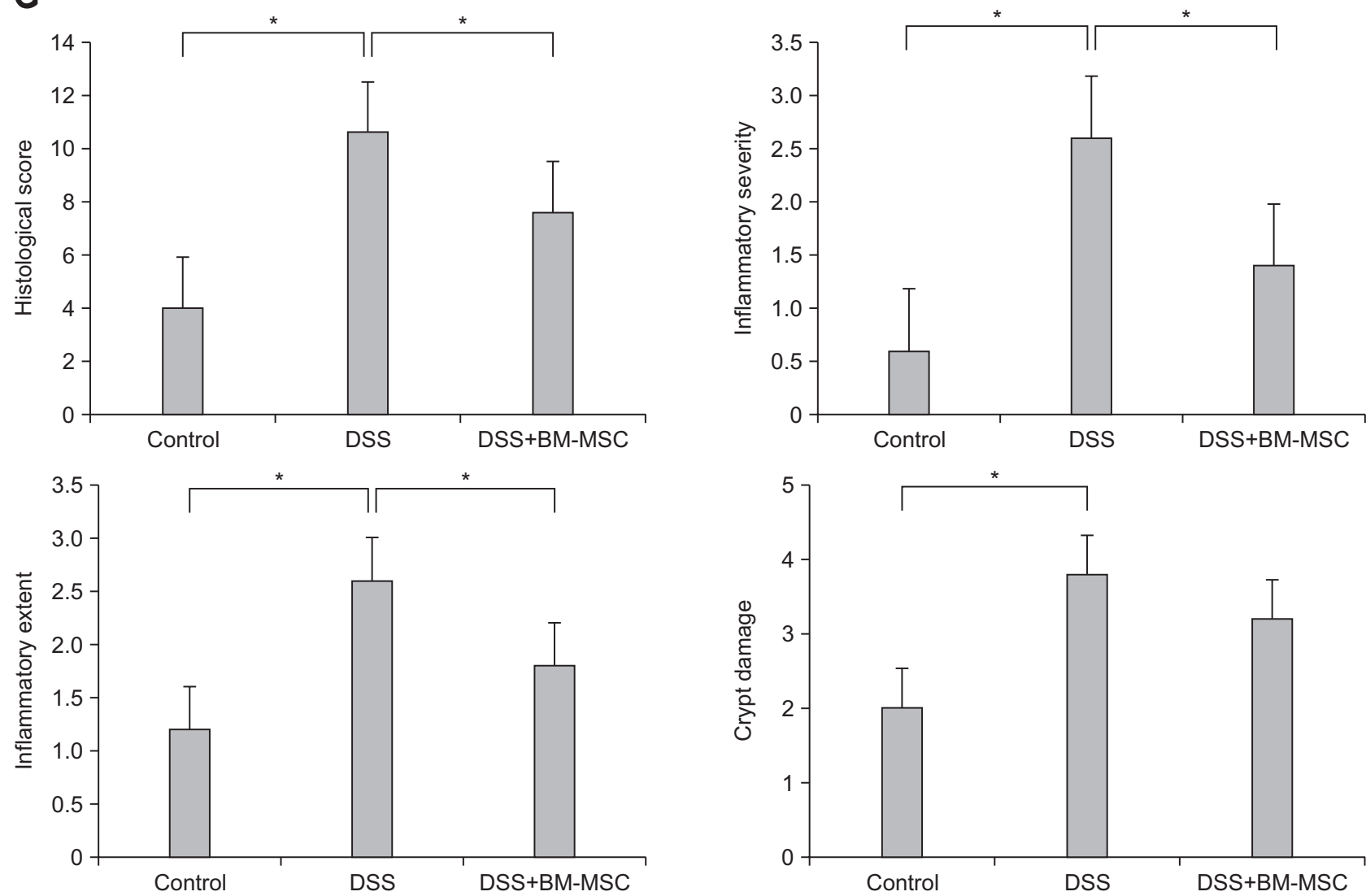

Fig. 3. Histological improvement in bone marrow-derived mesenchymal stem cells (BM-MSCs)-transplanted, dextran-sulfate sodium (DSS)-induced chronic colitis. (A-F) Histological analysis was performed with H\&E staining of colon tissues. (G) In the BM-MSC-treated, DSS-induced colitis group, total histological scores were significantly reduced compared with the DSS-only-treated group (control, untreated group 4.0 \pm 1.9 ; $3 \%$ DSStreated group 10.6 $\pm 2.3 ; 3 \%$ DSS+BM-MSC-treated group $\left.7.6 \pm 1.3 ;{ }^{*} \mathrm{p}<0.05\right)$. The individual scores, including inflammatory severity, extent, and crypt damage, were also decreased with BM-MSC treatment compared with the scores of the DSS-only-treated group. The data are presented as the mean \pm standard error of mean. Representative histologic images are shown for the control group: A $(\times 100)$ and D $(\times 200)$; the 3\% DSS-treated group: B ( $\times 100)$ and E (x200); and the 3\% DSS+BM-MSC-treated group: C $(\times 100)$ and F $(\times 200)$. 
A

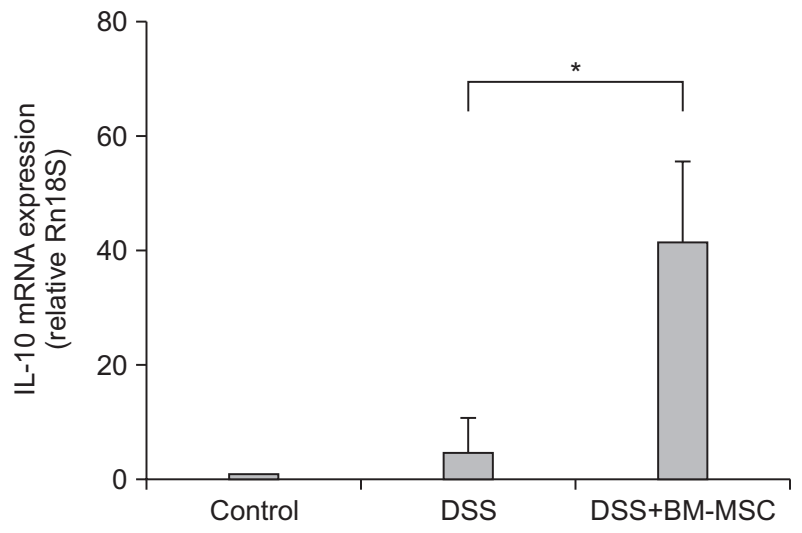

C

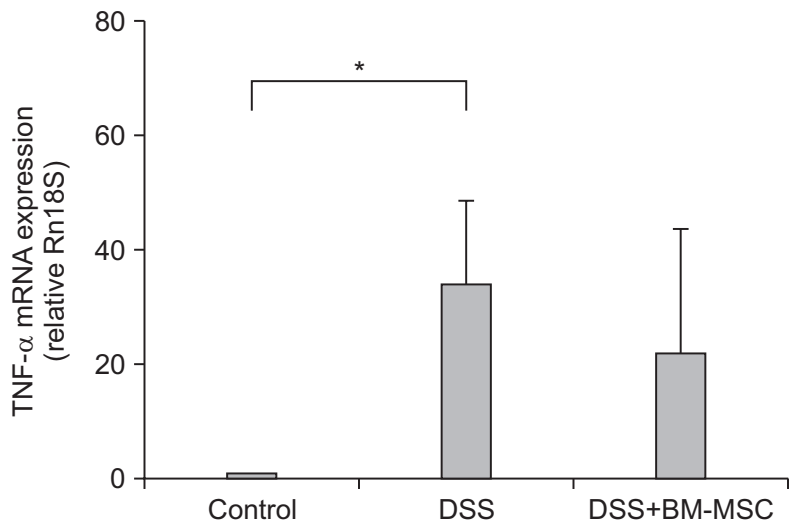

be determined. Furthermore, most of the studies focused on the prevention and improvement of acute gut inflammation by BMMSCs treatment. ${ }^{17,28,29}$ Therefore, in this study, we investigated the long-term effect of BM-MSCs specifically in chronic colitis induced by repeated DSS administration, which is an established experimental model for human IBD. ${ }^{30}$

We found long-term beneficial effects of BM-MSCs on clinicopathologic colitis activity in our chronic colitis model. In addition, with regard to the course of weight change, we found a greater preventive effect on weight loss in the second cycle of DSS administration and a more rapid restoring effect (without a preventive effect) in third cycle. These results suggest a decreasing beneficial effect of BM-MSC treatment with the progression of time.

In regard to the potential mechanisms for the therapeutic effect of BM-MSCs suggested by experimental models of IBD, BM-MSCs have been reported to downregulate the expression of Th1-type cytokines ${ }^{15,17,28,29}$ and upregulate the population of FoxP3 ${ }^{+}$regulatory $\mathrm{T}$ cells, with a consequent increase in IL10 production..$^{15,28,29,31}$ In agreement with previous studies, we showed a significant increase in IL-10 production in BM-MSCtreated mice. IL-10, which is a signature cytokine for a subset of CD4 T cells that exhibit regulatory functions, induces Treg populations and attenuates experimental colitis. ${ }^{23,24}$ IL-10-se-

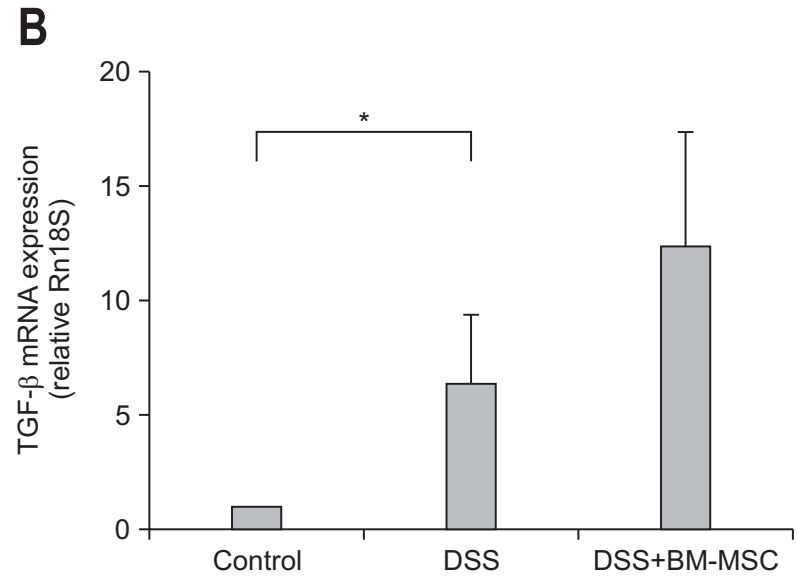

Fig. 4. Effect of bone marrow-derived mesenchymal stem cells (BMMSCs) on the production of anti-inflammatory cytokines (IL-10 and TGF- $\beta$ ) (A, B) and pro-inflammatory cytokine, TNF- $\alpha$ (C). mRNA expression of inflammatory mediators was measured in colonic tissue samples by using real-time polymerase chain reaction. BM-MSC treatment reduced the mRNA expression of tumor necrosis factor $\alpha$ and increased the mRNA expression of interleukin-10 and transforming growth factor $\beta$. Similar results were obtained from three independent experiments. The data are presented as the mean \pm standard error of mean ( $\left.{ }^{*} \mathrm{p}<0.05\right)$.

DSS, dextran-sulfate sodium.

creting Treg cells are known to play a critical role in maintaining intestinal homeostasis by suppressing immune response to resident commensal microbes. ${ }^{32}$ In addition, our results revealed that TGF- $\beta$ levels also were increased in DSS-induced colitis mice that were treated with BM-MSCs, suggesting the possibility of FoxP3 ${ }^{+}$regulatory $\mathrm{T}$ cell induction ${ }^{23,24}$ and contribution to the intestinal repair process. ${ }^{16}$

However, compared with previous studies that indicate BMMSC treatment downregulates the Th1-driven inflammatory responses in DSS-induced colitis mice, ${ }^{15,17}$ our data did not show a significant decrease in TNF- $\alpha$ levels upon BM-MSC transplantation. DSS administration in the present study, as in other recent studies, ${ }^{15,17,28,29}$ increased the proinflammatory cytokine of TNF- $\alpha$ in inflamed mucosa, verifying the crucial role of TNF- $\alpha$ in the development of IBD. ${ }^{2,3}$ The lack of a definite inhibitory effect of Th1 response in BM-MSC-treated mice of our experiment might be attributable to the change of cytokine profiles according to the acute or chronic stage of colitis. DSS-induced colitis is known to switch from Th1-Th17-mediated acute inflammation to a predominant Th2-mediated inflammatory response in the chronic state. ${ }^{33}$ In addition, because we used a chronic DSSinduced colitis model to evaluate the long-term effect of BMMSCs, the recoverable extent of TNF- $\alpha$ might be lower than that expected in acute colitis and, therefore, we could not observe 
significant suppressive effect of TNF- $\alpha$ expression after BMMSCs treatment. No significant improvement in colon length in our experiment could be also explained by chronic repeated injury by three cycles of DSS administration, which could contribute to unrecoverable chronic injury like fibrosis.

The mechanism of the long-term effect of BM-MSCs is not likely due to long-term survival of injected BM-MSCs because we found no GFP positive cells in BM-MSCs-treated mice in our experiments and previous other studies demonstrate a rapid disappearance of injected MSCs. ${ }^{17,28,29}$ However, given the reported long survival of T cells in peripheral tissues, ${ }^{34}$ long survival of regulatory $\mathrm{T}$ cells induced by initial injections of BM-MSCs could contribute to the long-term beneficial effect of BM-MSCs. Additional detailed experiments regarding the fate of BM-MSCinduced regulated $\mathrm{T}$ cell population are needed to confirm this possibility.

In our study, we used chemically induced colitis model by DSS administration. Because acute, chronic, or relapsing model can be produced easily by changing the concentration of DSS, it is one of the most commonly used animal models for studying IBD. ${ }^{33,35}$ However, as other animal models of genetically modified and adoptive transfer models, none of these models could represent the complexity of human disease. In addition, to date, there have been many experimental studies of the effects of MSCs in colitis models. However, we need more data about quality control of MSCs, different effects by different cell sources, and effective dose, frequency, and route based on the duration of effect for clinical application. Our data could give some information on the effect of MSCs with the progression of time.

In conclusion, we demonstrated that BM-MSC transplantation could be an effective means to treat chronic colitis in mice. In particular, initial systemic infusion of BM-MSCs at disease onset could exert preventive and rapid recovering effects through long-term immunosuppressive action in repeated DSS-treated chronic colitis.

\section{CONFLICTS OF INTEREST}

No potential conflict of interest relevant to this article was reported.

\section{ACKNOWLEDGEMENTS}

This study was supported by a grant of the Korea Healthcare technology R\&D Project, Ministry for Health, Welfare \& Family Affairs, Republic of Korea (A090898).

\section{REFERENCES}

1. Xavier RJ, Podolsky DK. Unravelling the pathogenesis of inflammatory bowel disease. Nature 2007;448:427-434.
2. Baumgart DC, Carding SR. Inflammatory bowel disease: cause and immunobiology. Lancet 2007;369:1627-1640.

3. Bouma G, Strober W. The immunological and genetic basis of inflammatory bowel disease. Nat Rev Immunol 2003;3:521-533.

4. Baumgart DC, Sandborn WJ. Inflammatory bowel disease: clinical aspects and established and evolving therapies. Lancet 2007;369:1641-1657.

5. Torres J, Danese S, Colombel JF. New therapeutic avenues in ulcerative colitis: thinking out of the box. Gut 2013;62:1642-1652.

6. Vermeire S, Ferrante M, Rutgeerts P. Recent advances: personalised use of current Crohn's disease therapeutic options. Gut 2013;62:1511-1515.

7. Drakos PE, Nagler A, Or R. Case of Crohn's disease in bone marrow transplantation. Am J Hematol 1993;43:157-158.

8. Kim TI. Clinical trials with stem cells in digestive diseases and future perspectives. Korean J Gastroenterol 2011;58:139-143.

9. Phinney DG, Prockop DJ. Concise review: mesenchymal stem/ multipotent stromal cells. The state of transdifferentiation and modes of tissue repair: current views. Stem Cells 2007;25:28962902.

10. De Miguel MP, Fuentes-Julián S, Blázquez-Martínez A, et al. Immunosuppressive properties of mesenchymal stem cells: advances and applications. Curr Mol Med 2012;12:574-591.

11. Song IH, Jang BI. Stem cells in inflammatory bowel disease: new potential therapeutic target. Intest Res 2013;11:79-84.

12. García-Bosch 0, Ricart E, Panés J. Review article: stem cell therapies for inflammatory bowel disease: efficacy and safety. Aliment Pharmacol Ther 2010;32:939-952.

13. Le Blanc K, Rasmusson I, Sundberg B, et al. Treatment of severe acute graft-versus-host disease with third party haploidentical mesenchymal stem cells. Lancet 2004;363:1439-1441.

14. Fang B, Song YP, Liao LM, Han Q, Zhao RC. Treatment of severe therapy-resistant acute graft-versus-host disease with human adipose tissue-derived mesenchymal stem cells. Bone Marrow Transplant 2006;38:389-390.

15. Gonzalez-Rey E, Anderson P, González MA, Rico L, Büscher D, Delgado M. Human adult stem cells derived from adipose tissue protect against experimental colitis and sepsis. Gut 2009;58:929939.

16. Tanaka H, Arimura Y, Yabana T, et al. Myogenic lineage differentiated mesenchymal stem cells enhance recovery from dextran sulfate sodium-induced colitis in the rat. J Gastroenterol 2011;46: 143-152.

17. He XW, He XS, Lian L, Wu XJ, Lan P. Systemic infusion of bone marrow-derived mesenchymal stem cells for treatment of experimental colitis in mice. Dig Dis Sci 2012;57:3136-3144.

18. Garcia-Olmo D, Herreros D, Pascual I, et al. Expanded adiposederived stem cells for the treatment of complex perianal fistula: a phase II clinical trial. Dis Colon Rectum 2009;52:79-86.

19. Duijvestein M, Vos AC, Roelofs H, et al. Autologous bone marrowderived mesenchymal stromal cell treatment for refractory luminal Crohn's disease: results of a phase I study. Gut 2010;59:1662- 
1669.

20. Bruder SP, Kurth AA, Shea M, Hayes WC, Jaiswal N, Kadiyala S. Bone regeneration by implantation of purified, culture-expanded human mesenchymal stem cells. J Orthop Res 1998;16:155-162.

21. Whittem CG, Williams AD, Williams CS. Murine colitis modeling using dextran sulfate sodium (DSS). J Vis Exp 2010;(35):e1652.

22. Williams KL, Fuller CR, Dieleman LA, et al. Enhanced survival and mucosal repair after dextran sodium sulfate-induced colitis in transgenic mice that overexpress growth hormone. Gastroenterology 2001;120:925-937.

23. Groux H, O'Garra A, Bigler M, et al. A CD4+ T-cell subset inhibits antigen-specific T-cell responses and prevents colitis. Nature 1997;389:737-742.

24. Boden EK, Snapper SB. Regulatory T cells in inflammatory bowel disease. Curr Opin Gastroenterol 2008;24:733-741.

25. Tse WT, Pendleton JD, Beyer WM, Egalka MC, Guinan EC. Suppression of allogeneic T-cell proliferation by human marrow stromal cells: implications in transplantation. Transplantation 2003; 75:389-397.

26. Prevosto C, Zancolli M, Canevali P, Zocchi MR, Poggi A. Generation of CD4+ or CD8+ regulatory T cells upon mesenchymal stem cell-lymphocyte interaction. Haematologica 2007;92:881-888.

27. Strober W, Fuss IJ. Proinflammatory cytokines in the pathogenesis of inflammatory bowel diseases. Gastroenterology 2011;140:17561767.
28. González MA, Gonzalez-Rey E, Rico L, Büscher D, Delgado M. Adipose-derived mesenchymal stem cells alleviate experimental colitis by inhibiting inflammatory and autoimmune responses. Gastroenterology 2009;136:978-989.

29. Castelo-Branco MT, Soares ID, Lopes DV, et al. Intraperitoneal but not intravenous cryopreserved mesenchymal stromal cells home to the inflamed colon and ameliorate experimental colitis. PLoS One 2012;7:e33360.

30. Strober W, Fuss IJ, Blumberg RS. The immunology of mucosal models of inflammation. Annu Rev Immunol 2002;20:495-549.

31. Parekkadan B, Upadhyay R, Dunham J, et al. Bone marrow stromal cell transplants prevent experimental enterocolitis and require host CD11b+ splenocytes. Gastroenterology 2011;140:966-975.

32. Izcue A, Coombes JL, Powrie F. Regulatory T cells suppress systemic and mucosal immune activation to control intestinal inflammation. Immunol Rev 2006;212:256-271.

33. Alex P, Zachos NC, Nguyen T, et al. Distinct cytokine patterns identified from multiplex profiles of murine DSS and TNBSinduced colitis. Inflamm Bowel Dis 2009;15:341-352.

34. Nguyen VH, Zeiser R, Dasilva DL, et al. In vivo dynamics of regulatory T-cell trafficking and survival predict effective strategies to control graft-versus-host disease following allogeneic transplantation. Blood 2007;109:2649-2656.

35. Perše M, Cerar A. Dextran sodium sulphate colitis mouse model: traps and tricks. J Biomed Biotechnol 2012;2012:718617. 